\title{
La France dans le Pacifique Sud. Les enjeux de la puissancedeNathalie Mrgudovic
}

\section{Raymond MAYER}

\section{(2) OpenEdition}

\section{Journals}

Édition électronique

URL : http://journals.openedition.org/jso/6164

DOI : $10.4000 /$ jso.6164

ISSN : $1760-7256$

Éditeur

Société des océanistes

\section{Édition imprimée}

Date de publication : 15 décembre 2010

Pagination : 257-258

ISBN : 978-2-85430-027-7

ISSN : 0300-953x

Référence électronique

Raymond MAYER, "La France dans le Pacifique Sud. Les enjeux de la puissancedeNathalie Mrgudovic », Journal de la Société des Océanistes [En ligne], 130-131 | 2010, mis en ligne le 15 décembre 2010, consulté le 21 septembre 2020. URL : http://journals.openedition.org/jso/6164 ; DOI : https://doi.org/ $10.4000 / j s 0.6164$ 
Mrgudovic Nathalie, 2009, La France dans le Pacifique Sud. Les enjeux de la puissance, Paris, L'Harmattan, 437 p., préface de Michel Rocard, bibliogr., index.

Il ne suffit sans doute pas d'une préface d'un ancien Premier ministre de la République française pour avaliser le contenu d'une thèse de doctorat de science politique. Mais la signature de Michel Rocard apporte indéniablement à ce travail universitaire un label qui souligne l'intérêt qu'un chef de gouvernement des années 1988-1993 y a pris et le rôle qu'il a personnellement joué sur le terrain géopolitique océanien pendant cette période. La thèse du livre se rapporte précisément au renversement de positionnement de la France dans le Pacifique Sud examiné sur une période de quarante ans, de 1966 à 2006. Nathalie Mrgudovic s'est proposé, dans le cadre de cette thèse soutenue à l'IEP de Bordeaux, de refaire l'histoire des relations diplomatiques de la France en Océanie pendant une période cruciale d'essais atomiques, en étudiant de manière systématique, tout en suivant un ordre grosso modo chronologique, les différentes phases de l'inversion de tendance en faveur de la France. Au-delà du signalement des péripéties qui l'ont marquée, l'auteur y exerce, comme il se doit, ses talents d'analyste politique.

Le plan de la thèse obéit au principe diachronique et à celui d'un découpage standardisé (mais toujours efficace) en trois étapes. Et, suivant un autre principe bien établi dans la science historique, chaque étape se voit assigner un intitulé thématique qui est censé en définir l'essentiel du vécu historique de l'époque, le tout dans une posture a posteriori qui fait les délices des sciences humaines! Passons!

La première partie est ainsi thématiquement lue comme celle des «Fondements de la présence française dans le Pacifique Sud », la deuxième comme celle des « Enjeux de la contestation régionale », et la troisième comme celle d' "Une puissance acceptée ». Bref le renversement en trois actes, le tout orchestré sous la baguette démiurgique d'un Premier ministre de République française.

Chaque partie est « travaillée » en deux chapitres, ce qui donne une ossature d'argumentaire un peu carrée, mais destinée à susciter l'adhésion unanime d'un jury de thèse normalement constitué. Évidemment, et c'est le propre de toute thèse qui souffre d'engendrer une antithèse, on pourrait contester le concept de « fondement » comme légitimation de la présence annexionniste d'un État tiers dans un jeu de quilles océanien. L'histoire ne saurait «fonder» aucune légitimité, quelle qu'elle soit. Cela dit, on se rappellera avec l'auteur de la thèse que le Pacifique fut, comme d'autres continents, l'enjeu de puissances coloniales rivales, mais la « rivalité contrainte » (p. 68) n'excuse pas l'absence de « grand dessein » (p. 69). Le deuxième chapitre de la première partie est entièrement consacré à l'intérêt nucléaire qui s'est historiquement dessiné dans le Pacifique, à la suite de la " perte » de l'Algérie par le pavillon tricolore. La doctrine de la « dissuasion » est bien récapitulée dans ses arguments dirimants à l'époque, mais susceptibles d'être mis en doute à une époque ultérieure, en fonction de l'évolution de la situation politique mondiale et des blocs stratégiques en présence.

La deuxième partie nous replonge à l'époque où l'on pouvait lire sous les ponts de chemins de fer néo-zélandais « French go home », faisant alors écho aux « US go home » qui fleurissaient dans les espaces publics de l'Hexagone vivant à l'heure de la guerre du Vietnam. Dans un classement des faits chapitré en deux catégories, celle de l'homogénéité et celle de l'hétérogénéité, l'auteur détaille dans sa deuxième partie de thèse les diverses phases de la contestation généralisée qui a marqué les années 1970 à 1990 contre la France, en notant le paroxysme qui semble avoir été atteint autour de la dernière " campagne » d'essais nucléaires français sous le premier mandat présidentiel de Jacques Chirac. Indépendamment de l'artifice de la forme, on pourra, là aussi, ergoter sur des expressions un peu dilettantes du traitement du Pacifique Sud comme d'un " lac nucléaire » sur l'air de " mare nostrum », ou de la Polynésie comme dernier " désert» français. Mais on prendra un intérêt certain à lire l'analyse de Nathalie Mrgudovic sur la montée en puissance du droit international de la mer et sur les conséquences connues de ce nouvel état de fait dans le Pacifique.

Sur ce thème précis du droit de la mer (pp. 86-99), l'auteur développe le rôle prééminent exercé par le gouvernement des Fidji (p. 88-90) pour faire aboutir cette idée qui étend d'un coup les espaces maritimes d'une terre de $19103 \mathrm{~km}^{2}$ (la Nouvelle-Calédonie) à $1740000 \mathrm{~km}^{2}$, de $3521 \mathrm{~km}^{2}$ (la Polynésie française) à $5030000 \mathrm{~km}^{2}$, ou encore de $255 \mathrm{~km}^{2}$ (Wallis-etFutuna) à $271050 \mathrm{~km}^{2}$, soit la moitié du territoire métropolitain! Un tableau récapitule toutes les données utiles en cette matière. Il est signalé aussi que des États importants (USA par exemple) n'ont pas encore ratifié le traité, et que d'autres ont émis des restrictions juridiques à son application. Un traité vaut ce que valent les intentions réelles de ses signataires, indépendamment des textes strictement signés !

La troisième partie passe au peigne fin l'heureuse transformation des rapports franco-océaniens à partir de la décennie 1990. À ce moment, l'action personnelle du Premier ministre Rocard a indubitablement fait retomber les effets du dramatique dérapage institutionnel engendré par les « événements » de NouvelleCalédonie, mais c'est surtout la fin déclarée et réalisée des essais nucléaires qui amène un nouveau deal dans les relations franco-océaniennes. Derrière le constat primaire, il est effectivement intéressant de faire l'analyse historique détaillée des processus qui ont généré une nouvelle répartition des accords entre puissances riveraines et puissances extérieures. De ce point de vue, la thèse de Nathalie Mrgudovic a tout le mérite d'exister, ne serait-ce que comme capacité critique vis-à-vis de décisions politiques, qui ne sont souvent appréhendées dans leur contexte historique que longtemps après les faits.

Je voudrais aussi souligner l'intérêt tout particulier des douze annexes qui procurent une documentation riche et abondante concernant non seulement les essais atomiques français, mais également sur l'évolu- 
tion statutaire des territoires du Pacifique accédant progressivement à leur souveraineté internationale, pendant la période incriminée. Sur le premier thème, on a droit au schéma type d'un forage souterrain pour y tester les effets d'une explosion nucléaire, on a le tableau récapitulatif de tous les essais conduits sur les sites de Mururoa et Tangafua, et la carte des zones censément dénucléarisées de la planète après les traités successifs de l'Antarctique, de Tlatelolco, de Rarotonga, de Bangkok et de Pelindaba. Sur le second thème, on découvrira la composition et l'année d'adhésion des territoires (indépendants ou non) à la Commission du Pacifique Sud, de même au Forum des Iles, et une mise à jour des statuts souvent complexes (e.g. the United States Unincorporated Territory of Guam) de ces mêmes territoires. Bref, l'intérêt du livre est non seulement au cœur de son développement, mais aussi en marge du débat principal.

Entre l'ouvrage d'Alain Babadzan qui est recensé ici et celui de Nathalie Mrgudovic, on ne peut manquer de saisir la différence entre le traitement anthropologique d'un ensemble géopolitique et son traitement correspondant en science politique. On a d'un côté une multilatéralité de perspectives qui se trouve développée sur le thème en présence, et de l'autre un regard scientifiquement focalisé sur une unilatéralité de perspective. Les deux approches sont également respectables et il n'est pas question pour moi de renier la fécondité de l'une et l'autre démarches, mais il est clair que l'on peut se sentir à l'étroit dans une perspective par trop « nationale ».

Raymond MAYER, Université Omar Bongo de Libreville et Université de Lyon 2

Gossler Claus, 2006. Die Société commerciale de l'Océanie (1876-1914). Aufstieg und Untergang der Hamburger Godeffroys in Ost-Polynesien, Bremen, MontAurum Verlag, 592 p., bibliogr., annexes, 28 ill. noir et blanc, 6 cartes.

Pour ceux des océanistes qui le connaissent, le nom de Godeffroy évoque avant tout la firme hambourgeoise qui fut la première à investir durablement et massivement le Pacifique, entre l'installation d'un comptoir à Apia (Samoa) en 1857 et la faillite de Godeffroy \& Sohn en 1879, peu avant les négociations diplomatiques d'où allait émerger l'empire colonial allemand dans le Pacifique, dont cette firme avait fondé les premiers postes avancés. Ce nom peut aussi leur rappeler le musée privé fondé à Hambourg par Johann Cesar vi Godeffroy, fourni en « curiosités » de tous ordres, " artificielles » autant que « naturelles », par les capitaines de ses bateaux, afin de présenter au public « une image complète » des mers du Sud, et qui rassembla, selon l'appréciation d'Adolf Bastian, directeur du musée ethnographique de Berlin, « une des plus magnifiques collections en ce domaine». Après la faillite de 1879 , cette collection fut vendue par lots à des musées allemands, selon leurs moyens respectifs ou l'intérêt qu'ils y portaient (voir le travail
d'H. Glenn Penny, absent de la bibliographie de Cl. Gossler), le musée de Leipzig finissant d'acquérir tout ce qui en subsistait après la mort en 1885 de Johann Cesar vi.

Or le neveu de celui-ci, Gustav Godeffroy Jr, avait trouvé à s'établir à Tahiti, à s'y marier avec une des filles Brander et à développer en association avec d'autres une firme qui se maintint pendant près de quarante ans, la Société Commerciale de l'Océanie (SCO). C'est l'histoire de ce « joint-venture » paradoxal que Claus Gossler étudie ici, avec maints autres sujets, sous un titre franco-allemand propre à susciter l'étonnement et la curiosité. L'ouvrage (dont la rédaction du Jso n'a appris l'existence que tardivement) est luimême d'aspect austère, imprimé en petits corps avec des marges réduites au minimum et plus de 2500 notes infrapaginales. Cette mise en pages ponctuée d'illustrations souvent illisibles souligne peut-être (mais dessert surtout) la minutie d'analyse et l'ampleur exceptionnelle de la documentation de cette dissertation doctorale récompensée par l'université de Hambourg en 2005. Le volume est dépourvu d'index, mais sa table des matières très détaillée permet de repérer sans peine les principaux thèmes et protagonistes de cette somme, qui excède largement le seul domaine des «sciences économiques et sociales » (ou de « l'histoire des entreprises » ainsi qu'écrit l'auteur p. 15) pour déboucher sur un impressionnant tableau d'histoire « totale ».

Issu lui-même d'une vieille famille de négociants hambourgeois, Claus Gossler a eu la fortune, au terme d'une longue carrière dans le commerce international, de se voir ouvrir les archives familiales d'un ami (arrière petit-fils de Gustav Jr) et d'y découvrir les activités de la sCO, que mentionnaient certes les ouvrages consacrés à l'histoire de Tahiti qu'il consulta ensuite, mais sans en suggérer l'importance. Il s'est alors lancé dans l'exploration et l'exploitation des archives de la firme, conservées principalement au Staatsarchiv der Freien und Hansestadt Hamburg, complétées de multiples lectures pour en restituer le contexte et la portée. D'autres chercheurs dépourvus de l'expérience professionnelle, de l'« intelligence des affaires " et du sens des "réalités économiques 》 acquis par l'auteur auraient-ils su aussi bien mettre en œuvre ces matériaux ? Il était non seulement le mieux à même d'en tirer un parti utile à tous, mais il est allé très au-delà de cet objectif, et il est significatif que les recensions parues jusqu'à présent n'aient trouvé à reprocher à ce livre, du point de vue de l'histoire générale du Pacifique, qu'un minime anachronisme («Samoa occidentales» pp. 23 et 43, au lieu de «Samoa indépendantes »). On pourrait y ajouter quelques inadvertances, très peu nombreuses et presque inévitables dans un travail de cette ampleur (ainsi de la ligne « Neukaledonien » inutilement répétée dans le tableau de la p. 228). D'autres critiques trouveront peut-être à se préciser quand les lecteurs en sauront autant ou plus que l'auteur, mais ce n'est pas pour demain.

Après une introduction et des considérations de méthode (ch. 1), l'ouvrage étudie la création de la SCO à partir de l'Allemagne et des Samoa (ch. 2), la zone d'intérêt économique qu'elle s'arroge (ch. 3, extraor- 